\title{
ON ANALYTIC INJECTIVE MATRIX FUNCTIONS
}

\author{
D. BSHOUTY AND W. HENGARTNER
}

\begin{abstract}
An ingenious generalization of univalent functions of one complex variable into matrix functions was introduced by $B$. Schwarz, along with two conjectures corresponding to the first coefficient problem and the second coefficient problem for the traditional classes $\Sigma$ and $S$. respectively. We exhibit two counterexamples for these conjectures.
\end{abstract}

I. Introduction. Injective vector and matrix functions are defined as follows [1]. Let the (column) vector $u(z)=\left(u_{1}(z), \ldots, u_{n}(z)\right)^{T}$ be holomorphic in a domain $B$ of the $z$-plane (i.e. each component $u_{k}(z)$ is holomorphic in $\left.B\right) . u(z)$ is called injective in $B$ if $u\left(z_{1}\right) \neq u\left(z_{2}\right), z_{1}, z_{2} \in B, z_{1} \neq z_{2}$. An $n \times n$ matrix $U(z)=\left(u_{i k}(z)\right)_{1}^{n}$, holomorphic in $B$, is called injective there if for every constant vector $c, c \neq 0$, the holomorphic vector function $u(z)=U(z) c$ is injective in $B$. The following criterion for matrix injectivity is due to Schwarz [1].

$U(z)=\left(u_{i k}(z)\right)_{1}^{n}$ is injective in a domain $B$ if and only if all $u_{i k}(z)$ are analytic for all $z$ in $B$ and, moreover,

$$
L(z, \zeta)=\operatorname{det}((U(z)-U(\zeta)) /(z-\zeta)) \neq 0 \quad \text { for } z \in B, \zeta \in B
$$

with the obvious definition for $z=\zeta$. Let the class $S^{(n)}$ consist of all injective functions $V(z)$ in $D \equiv\{|z|<1\}$ with an expansion

$$
V(z)=I_{n} \cdot z+V_{2} \cdot z^{2}+\cdots \quad \text { in } D,
$$

where $I_{n}$ denotes the identity matrix of order $n$ and $V_{i}$ are $n \times n$ constant matrices. On the other hand the class $\Sigma^{(n)}$ consists of all injective functions $W(z)$ in $\Delta \equiv\{|z|>1\}$ such that

$$
W(z)=I_{n} \cdot z+W_{0}+W_{1} \cdot 1 / z+\cdots \quad \text { in } \Delta,
$$

where $W_{i}$ are constant matrices as the $V_{i}$ above. For $n=1$ these classes reduce to the traditional $S$ and $\Sigma$ classes. We remark that for a triangular matrix function $U(z)$ to be in $S^{(n)}$ it is necessary and sufficient that $u_{i i}(z)$ be in $S$ for all $i$.

For a square matrix $A$, let $\rho(A)$ denote the spectral radius of $A$. In [1] the following conjectures were introduced.

Conjecture 1. If $V(z)$ is in $S^{(n)}$, then $\rho\left(V_{2}\right) \leqslant 2$.

Conjecture 2. If $W(z)$ is in $\Sigma^{(n)}$, then $\rho\left(W_{1}\right) \leqslant 1$.

Received by the editors June 30, 1982 and, in revised form, October 12, 1982.

1980 Mathematics Subject Classification. Primary 30C55.

Key words and phrases. Univalent functions, injective matrix functions.

(C)1983 American Mathematical Society 0002-9939/83/0000-1163/\$02.00 
II. Counterexamples. We start by noting that it will be enough to construct the counterexamples for $n=2$. Indeed, if $A_{2}(z)$ is a counterexample for $n=2$, then the direct sum $A_{n}(z)=A_{2}(z) \oplus z \cdot I_{n-2}$ is a counterexample for arbitrary $n \geqslant 2$, as the corresponding $L($,$) and \rho($ ) are left unchanged.

We next note that the validity of Conjecture 1 for $n=2$ would imply the existence of many extremal functions. Indeed, as already remarked, for arbitrary $f(z)$ in $S$ and $g(z)=a_{2} z^{2}+\cdots$ analytic in $D$,

$$
V(z)=\left(\begin{array}{cc}
z /(1-z)^{2} & 0 \\
g(z) & f(z)
\end{array}\right) \in S^{(2)}
$$

would be extremal for the coefficient problem. (Whereas in fact, for almost any $f(z)$, $V(z)$ is not locally extremal.) For our counterexample we have chosen $f(z)=z$ for simplicity.

Let $\varepsilon>0$ and define

$$
J^{\varepsilon}(z)=\left|\begin{array}{cc}
\frac{z-\varepsilon z^{2}}{(1-z)^{2}} & \varepsilon h_{2}(z) \frac{(1+z)}{(1-z)} \\
h_{1}(z) \frac{(1+z)}{(1-z)} & z
\end{array}\right|=I_{2} \cdot z+J_{2}^{*} \cdot z^{2}+\cdots
$$

where $h_{1}(z), h_{2}(z)$ are even polynomials with real coefficients to be chosen later. One can show that for "almost all" $h_{1}(z)$ there is an $h_{2}(z)$ such that for small $\varepsilon$, $J^{\varepsilon}(z)$ is injective with $\rho\left(J_{2}^{*}\right)>2$. For our counterexample, we will fix $h_{1}(z)$ and construct a corresponding $h_{2}(z)$.

Finally we introduce our counterexamples as

THEOREM 1. There exists a real number $\alpha$ and an even polynomial $h_{2}(z)=a_{2} z^{2}$ $+\cdots$ with real coefficients and an $\varepsilon_{0}$ such that for all $0<\varepsilon \leqslant \varepsilon_{0}$,

$$
\begin{aligned}
G^{\varepsilon}(z) & =\left(\begin{array}{cc}
\frac{z-\varepsilon z^{2}}{(1-z)^{2}} & \frac{\varepsilon h_{2}(z)(1+z)}{1-z} \\
\frac{\left(z^{2}+\alpha z^{4}\right)(1+z)}{1-z} & z
\end{array}\right) \\
& =I_{2} \cdot z+G_{2}^{\varepsilon} \cdot z^{2}+G_{3}^{\varepsilon} \cdot z^{2}+\cdots
\end{aligned}
$$

is in $S^{(2)}$ and $\rho\left(G_{2}\right)>2$.

THEOREM 2. If $V(z)=I_{n} \cdot z+V_{2} \cdot z^{2}+V_{3} \cdot z^{2}+\cdots$ is in $S^{(n)}$, then

$$
\operatorname{Inv}(V(1 / z))=I_{n} \cdot z-V_{2}+\left(V_{2}^{2}-V_{3}\right) \cdot 1 / z+\cdots
$$

is in $\Sigma^{(n)}$. In particular, $\operatorname{Inv}\left(G^{\varepsilon}(1 / z)\right)$ is in $\Sigma^{(2)}$ with $\rho\left(G_{2}^{2}-G_{3}\right)>1$. 
III. Proof of theorems. To prove Theorem 1 we consider

$$
\begin{aligned}
\operatorname{det}\left(\frac{G^{\varepsilon}(z)-G^{\varepsilon}(\alpha)}{z-\zeta}\right)= & \frac{1-z \zeta-\varepsilon(z+\zeta-2 z \zeta)}{(1-z)^{2}(1-\zeta)^{2}} \\
& -\varepsilon \prod_{i=1}^{2}\left(\frac{(1-\zeta)(1+z) h_{i}(z)-(1-z)(1+\zeta) h_{i}(\zeta)}{(z-\zeta)(1-z)(1-\zeta)}\right)
\end{aligned}
$$

which will be shown to be different from zero in $D^{2} \equiv\{|z|<1\} \times\{|\zeta|<1\}$. This is equivalent to saying that the polynomial in $z, \zeta$,

$$
\begin{aligned}
M(z, \zeta) \equiv & 1-z \zeta-\varepsilon(z+\zeta-2 z \zeta) \\
& -\varepsilon \prod_{i=1}^{2}\left(\frac{(1-\zeta)(1+z) h_{i}(z)-(1-z)(1+\zeta) h_{i}(\zeta)}{z-\zeta}\right) \\
\equiv & 1-z \zeta+\varepsilon E(z, \zeta) \neq 0
\end{aligned}
$$

in $D^{2}$.

Fix $\zeta, \frac{1}{2} \leqslant|\zeta| \leqslant 1$. For sufficiently small $\varepsilon$ independent of $\zeta$, the root $z=z(\varepsilon)$ of the above expression in $|z| \leqslant 3$ is unique, analytic in $\varepsilon$ and is given explicitly by $z=1 / \zeta+\varepsilon b_{1}+\varepsilon^{2} b_{2}+\cdots$, where

$$
\zeta \cdot b_{1}=(2-1 / \zeta-\zeta)-\left[h_{2}(1 / \zeta)+h_{2}(\zeta)\right]\left[h_{1}(1 / \zeta)+h_{1}(\zeta)\right]
$$

This is a simple consequence of Rouché's theorem and the implicit function theorem, noting that $E(z, \zeta)$ is bounded on $|z|=3$.

We then consider the expression $\zeta \cdot b_{1}$ for $\zeta=e^{i \theta}$.

$$
\zeta \cdot b_{1}=(2-2 \operatorname{Cos} \theta)-4 \operatorname{Re}\left\{h_{2}(\zeta)\right\} \cdot \operatorname{Re}\left\{h_{1}(\zeta)\right\} .
$$

Given $h_{1}(z)=z^{2}+\alpha z^{4}$ we wish to construct an $h_{2}(z)$ such that $\zeta \cdot b_{1}$ is strictly larger than zero for $\zeta=e^{i \theta}$. In fact, we construct an $h_{2}(z)$ as in the statement of the theorem with the property that $\operatorname{Re}\left\{h_{2}(\zeta)\right\} \operatorname{Re}\left\{h_{1}(\zeta)\right\} \leqslant 0, \zeta=e^{i \theta}$, and strictly less than zero for $\zeta=1$. To do so we proceed as follows. For an arbitrary Lebesgue integrable function $f(\theta)$ on $[-\pi, \pi]$, let

$$
c_{n}=\frac{1}{2 \pi} \int_{-\pi}^{\pi} f(\theta) e^{-i n \theta} d \theta, \quad n=0, \pm 1, \pm 2, \ldots,
$$

be its Fourier coefficients. Let $T_{k}=\left(c_{\nu-\mu}\right)_{0 \leqslant \mu, \nu \leqslant k}=0$ be its corresponding Toeplitz matrix of order $k$. Then for an arbitrary polynomial $P(z)=\sum_{n=0}^{k} u_{n} z^{n}$,

$$
\frac{1}{2 \pi} \int_{-\pi}^{\pi} f(\theta)\left|P\left(e^{i \theta}\right)\right|^{2} d \theta=\sum_{\nu, \mu=0}^{k} c_{\nu-\mu} \bar{u}_{\nu} u_{\mu} .
$$

We now define $h_{2}(z)$ by

$$
\operatorname{Re} h_{2}(\zeta)=-\operatorname{Re} h_{1}(\zeta) \cdot|P(\zeta)|^{2}, \quad \zeta=e^{i \theta},
$$

where $P(z)=u_{0}+u_{2} z^{2}+u_{4} z^{4}, u_{i}$ real. This defines $h_{2}(z)$ in $D$ uniquely if we assume $\operatorname{Im} h_{2}(0)=0$. The specific choice of $P(z)$ implies that

$$
h_{2}(z)=a_{0}+a_{2} z^{2}+a_{4} z^{4}+\cdots, \quad a_{i} \text { real, }
$$


and

$$
\begin{aligned}
a_{0} & =-\frac{1}{2 \pi} \int_{-\pi}^{\pi} \frac{h_{1}(\zeta)+\overline{h_{1}(\zeta)}}{2}|P(\zeta)|^{2} d \theta=-\left(u_{0} u_{2}+u_{2} u_{4}+\alpha u_{0} u_{4}\right) \\
a_{2} & =-\frac{1}{2 \pi} \int_{-\pi}^{\pi} \frac{h_{1}(\zeta)+\overline{h_{1}(\zeta)}}{2} \cdot e^{-2 i \theta}|P(\zeta)|^{2} d \theta \\
& =-\left(u_{0}^{2}+u_{2}^{2}+u_{4}^{2}\right)-\alpha\left(u_{0} u_{2}+u_{2} u_{4}\right)-u_{0} u_{4}
\end{aligned}
$$

Let $a_{0}=0$. Then $\rho\left(G_{2}^{\varepsilon}\right)=2+\left(a_{2}-2\right) \varepsilon / 2+O\left(\varepsilon^{2}\right)>2$ for $\varepsilon<\varepsilon_{1}$ if $a_{2}>2$. Any choice for $u_{i}$ with $a_{0}=0$ and $a_{2}>2$ will give the desired $h_{2}(z)$. We should only add the condition that $P(1)=u_{0}+u_{2}+u_{4} \neq 0$.

We now proceed to show that

$$
M(z, \zeta) \equiv 1-z \zeta+\varepsilon E(z, \zeta) \neq 0
$$

in $D^{2}$ for small values of $\varepsilon$. Let $m$ denote a bound for $|E(z, \zeta)|$ in $D^{2}$ and let $r_{0}<1$ be such that $\operatorname{Re}\left\{\zeta \cdot b_{1}\right\}>\delta>0$, for $1 \geqslant|\zeta| \geqslant r_{0}$, for sufficiently small $\delta$. These choices are possible since $\zeta \cdot b_{1}$ was found to be strictly positive on $|\zeta|=1$. We let $\varepsilon_{2}$ be such that for $1 \geqslant|\zeta| \geqslant r_{0}$ and $0<\varepsilon<\varepsilon_{2}$,

$$
|z \cdot \zeta|^{2}=\left|1+b_{1} \zeta \varepsilon+b_{2} \zeta \varepsilon^{2}+\cdots\right|^{2}>1,
$$

This is possible since $|z \cdot \zeta|^{2}=1+2 \operatorname{Re}\left\{b_{1} \cdot \zeta\right\} \varepsilon+O\left(\varepsilon^{2}\right)$. Define

$$
\varepsilon_{0}=\operatorname{Min}\left(\varepsilon_{1}, \varepsilon_{2},\left(1-r_{0}\right) / 2 m\right) \text {. }
$$

Then for $0<\varepsilon<\varepsilon_{0}$, (3) shows that $M(z, \zeta) \neq 0$ in $D_{z}$ if $1 \geqslant|\zeta| \geqslant r_{0}$, whereas for $|\zeta|<r_{0}$

$$
|M(z, \zeta)| \geqslant 1-r_{0}-\varepsilon_{0} m>\varepsilon_{0} m \text { in } D_{z} .
$$

This completes the proof of Theorem 1.

To prove Theorem 2, we remark that $\operatorname{det}(V(z) / z) \neq 0$. Thus

$$
W(z)=\operatorname{Inv}(V(1 / z))=(\operatorname{Adj} z V(1 / z)) / \operatorname{det}(z V(1 / z))
$$

is defined and analytic in $\Delta$. Moreover,

$$
z V\left(\frac{1}{z}\right) \cdot\left(\frac{W(z)-W(\zeta)}{z-\zeta}\right) \cdot \zeta V\left(\frac{1}{\zeta}\right)=\frac{V(1 / \zeta)-V(1 / z)}{(1 / \zeta)-(1 / z)} \text { for } z, \zeta \text { in } \Delta
$$

so that $\operatorname{det}((W(z)-W(\zeta)) /(z-\zeta)) \neq 0$, for $z, \zeta$ in $\Delta$, and the theorem is proved.

In particular,

$$
\begin{aligned}
G_{2}^{2}-G_{3} & =\left(\begin{array}{cc}
2-\varepsilon & \varepsilon a_{2} \\
1 & 0
\end{array}\right)^{2}-\left(\begin{array}{cc}
3-2 \varepsilon & 2 \varepsilon a_{2} \\
2 & 0
\end{array}\right) \\
& =\left(\begin{array}{cc}
1+\varepsilon\left(a_{2}-2\right) & 0 \\
-\varepsilon & \varepsilon a_{2}
\end{array}\right)+O\left(\varepsilon^{2}\right),
\end{aligned}
$$


so that $\rho\left(G_{2}^{2}-G_{3}\right)=1+\left(2 a_{2}-2\right) \varepsilon+O\left(\varepsilon^{2}\right)>1$ if $a_{2}>2$. One possible choice for $u_{i}$ that fits both cases is $u_{0}=u_{4}=1 ; u_{2}=-\alpha / 2 ; 16>\alpha^{2}>20 / 3$.

In view of the above discussion, we suggest for further investigation the following question that we were unable to settle:

Problem. Is it true or not that $\rho\left(V_{2}\right)$ or $\rho\left(W_{1}\right)$ is bounded?

\section{REFERENCES}

1. B. Schwarz, Injective matrix functions, Proc. Amer. Math. Soc. 83 (1981), 331-336.

Department of Mathematics, Technion-Israel Institute of Technology, Haifa, Israel

Department of Mathematics, Laval University, Quebec City, Quebec, Canada 